\title{
Functionalized Multi-walled Carbon Nanotubes as Emerging Carrier for Biological Applications
}

\author{
Ahmed A. Haroun ${ }^{1}$, Hanan M. Ahmed ${ }^{2}$, Eman F. Ahmed ${ }^{2}$ \\ ${ }^{1}$ Chemical Industries Research Division, National Research Centre \\ ElBohoth Str, Dokki, Giza, Egypt \\ aa.haroun@nrc.sci.eg; haroun68_2000@yahoo.com \\ ${ }^{2}$ Chemistry of Natural \& Microbial Products Dept, National Research Centre, \\ 12622 Dokki, Giza, Egypt.
}

\begin{abstract}
This work aims to bridge nanotechnology and biotechnology via comparative study of the influence of functionalized multi-walled carbon nanotubes (fMWCNTs) on some enzymes activity and structure by either covalent binding or adsorption techniques such as L-asparaginase (produced by Aspergillus versicolor, L-ASNase). The prepared materials were analyzed by scanning and transmission electron microscopes (SEM and TEM) and particle size distribution analysis using DLS technique. Besides, in vitro cytotoxicity of the prepared materials using SRB assay was carried out. The highest immobilization yield (\%) of L-ASNase was about $54 \%$ and the immobilized MWCNTs had particle size around 51$79 \mathrm{~nm}$ relative to the un-immobilized one $(180 \mathrm{~nm})$. Using of fMWCNTs for enzyme immobilization could be protecting the effect of elevating temperature at different period of time. Also, the storage time played a significant role in activation of the immobilized enzyme by releasing the embedded enzyme from fMWCNTs.
\end{abstract}

Keywords: Multi-walled carbon nanotubes, Bionanotechnology, Enzyme immobilization, In vitro cytotoxicity.

\section{Introduction}

Nanotechnology-inspired biocatalyst systems have attracted a lot of attention in enzyme immobilization recently. However, common immobilization methods have limited the applicability of these biocatalysts owing to enzyme leaching, 3D structure loss, strong diffusion resistance and expensive enzyme purification requirement before immobilization. Sitespecific enzyme immobilization method overcomes the foresaid limitations. This method does not require enzyme purification and the resulting nanoscale biocatalyst can maintain high enzyme activity and stability [1-3].

Enzyme immobilizations on carbon nanotubes (CNTs) for fabrication of biofuel cells and for preparation of biocatalysts are rapidly emerging as new research areas. Various immobilization methods have been developed, and in particular, specific attachment of enzymes on carbon nanotubes has been an important focus of attention. The method of immobilization has an effect on the preservation of the enzyme structure and retention of the native biological function of the enzyme [4,5]. Consequently, in this study characterization of the immobilized some of bioactive ingredients such as enzymes (L-ASNase ) onto fMWCNTs was investigated.

\section{Methods}

\subsection{Purification and oxidation of MWCNTs}

Pristine MWCNT (100 mg) was dispersed in mixed concentrated sulphuric and nitric acids $(3: 1, \mathrm{v} / \mathrm{v})$ at ratio of $50 \mathrm{~mL}$ acid mixture [6]. The resulted mixture was then heated at $110^{\circ} \mathrm{C}$ overnight with continuous stirring to produce oxidized carbon nanotubes (MWCNTs-COOH). The sample washed with ultrapure water until got neutral filtrate (pH 7.0). The collected solid was dried under vacuum at $70^{\circ} \mathrm{C}$ for $12 \mathrm{~h}$ and kept for further functionalization and analysis.

\subsection{Preparation of functionalized MWCNTs}

The MWCNTs functionalization was demonstrated using covalent or ionic binding processes in combination with sonication strategy [7,8]. MWCNTs-COOH powder (10mg) was dispersed in $200 \mathrm{~mL}$ HEPES then reacted with $100 \mathrm{mM}$ N- 
hydroxy succinimide (NHS) and 1-ethyl-3-[3-(dimethylamino) propyl] carbodiimide (EDC). N,N-bis(carboxymethyl) Llysine hydrate (ANTA) $(20 \mathrm{mM})$ was dissolved in buffer with excess cobalt chloride $\left(\mathrm{CoCl}_{2}\right)$ to form ANTA-Co ${ }^{2+}$. Excess $\mathrm{Co}^{2+}$ was precipitated by $\mathrm{NaOH}$. The resulting $\mathrm{Co}(\mathrm{OH})_{2}$ was removed by centrifugation at $8000 \mathrm{rpm}$ for $10 \mathrm{~min}$. The supernatant containing ANTA- $\mathrm{Co}^{2+}$ was collected then mixed with above MWCNTs solution to produce MWCNTs- ANTA$\mathrm{Co}^{2+}$ complex. The by-products and excess NHS were removed by washing the complex with $20 \mathrm{mM}$ buffer. The complex kept for further investigation.

\subsection{Enzyme immobilization}

L-ASNase immobilization was carried out using two different techniques:

Covalent binding technique:

The prepared complex, MWCNTs- ANTA-Co ${ }^{2+}$ as previously mentioned, was incubated with (3968 U/g carrier) of enzyme with (EDC) crosslinker at $4^{\circ} \mathrm{C}$ overnight and the resulting conjugate was collected by centrifugation. The immobilized enzyme formulation washed with buffer and kept for further analysis.

Simple physical adsorption technique:

Enzyme (3968 U/g carrier) was simple mixed in different ratios with oxidized MWCNTs aqueous solution without EDC crosslinker. The immobilized enzyme formulations were collected using centrifugation. The resulting samples washed with buffer and kept for further analysis.

\subsection{Enzyme activity [9]}

L-ASNase activity was determined in culture filtrates by quantifying the ammonia formation using Nessler's reagent, as shown in Fig 1. The enzymatic reaction mixture contains $0.5 \mathrm{ml}$ of $0.04 \mathrm{M}$ L-asparagine substrate, $0.5 \mathrm{ml}$ of $0.05 \mathrm{M}$ Tris$\mathrm{HCl}$ buffer ( $\mathrm{pH} 8.6$ ) and $0.5 \mathrm{ml}$ of crude enzyme. The enzyme substrate mixtures were incubated at $40^{\circ} \mathrm{C}$ for $30 \mathrm{~min}$, the enzyme activity was stopped by the addition of $0.5 \mathrm{ml}$ of $1.5 \mathrm{M}$ trichloroacetic acid (TCA). The liberated ammonia was coupled using Nessler's reagent for $15 \mathrm{~min}$ for development of colour. The optical density of the colour produced was measured at $450 \mathrm{~nm}$ using a double beam UV-visible spectrophotometer (ECIL model UV-5704 SS). The liberated ammonia was determined from the standard curve of ammonium sulphate. The L-ASNase activity was expressed in terms of International unit (IU). One unit (IU) of L-ASNase is defined as the amount of enzyme which liberates one $\mu \mathrm{moL}$ of ammonia per $\mathrm{mL}$ per minute $(\mu \mathrm{mole} / \mathrm{ml} / \mathrm{min})$ at $40^{\circ} \mathrm{C}$.

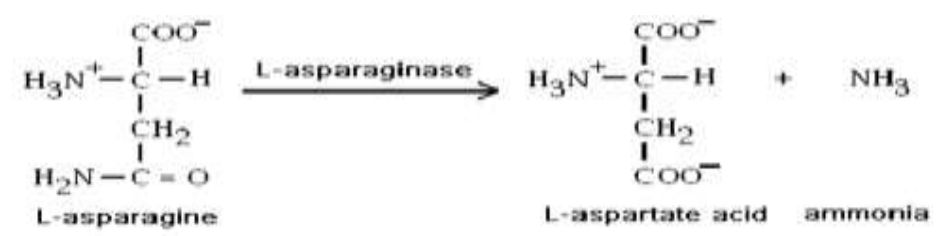

Fig. 1: Reaction of L-ASNase with substrate for liberating ammonia.

\subsubsection{Effect of storage time on the enzyme activity:}

The immobilized and L-ASNase free enzyme were mixed with the substrate and stored in the fridge at $4^{\circ} \mathrm{C}$ for different time periods (1, 2, 3 and 4 weeks), then the enzyme activity was determined.

\section{Results and Discussion}

The activity of the L-ASNase enzyme was assayed after 3 days of incubation. It was found that Aspergillus versicolor was the superior to produce the enzyme where the maximum activity $(45.53 \mathrm{U} / \mathrm{ml})$ was achieved after 3 days of incubation at $28{ }^{\circ} \mathrm{C}$. After further purification using $80 \%$ ethanol, the partial purified enzyme had a protein content of $12.075 \mathrm{mg}$ and specific activity $208.52 \mathrm{U} / \mathrm{mg}$ protein. Also, it had purification fold of 6.77 with enzyme recovery about $3.68 \%$. The partial purified enzyme was immobilized onto fMWCNTs with different ratios using covalent and physical adsorption techniques. The results confirmed that the highest immobilization yield $(54.0 \%)$ was obtained in case of ratio $(1: 1)$. While, the immobilization yield was $37.6 \%$ in case of ratio (1:2), using physical adsorption technique without EDC crosslinker. In other words, the physical absorption of the enzyme with fMWCNTs without the cross linker exhibited more efficient enzyme activity than that in case of the enzyme covalently bonded to the carrier. 
The immobilized L-ASNase formulations were stored with the substrate at $4^{\circ} \mathrm{C}$ for 4 weeks and the activity was calculated after each week. The results were presented in Fig 2. It was observed that the storage process played a significant role in activation of the immobilized enzyme according to the used technique. Using of covalent technique exhibited high enzyme activity after 3 week of storage (56.75) $\mathrm{U} / \mathrm{g}$ relative to zero time. While, in case of using physical technique, the activity of the immobilized enzyme was gradually decreased till reach about zero activity.

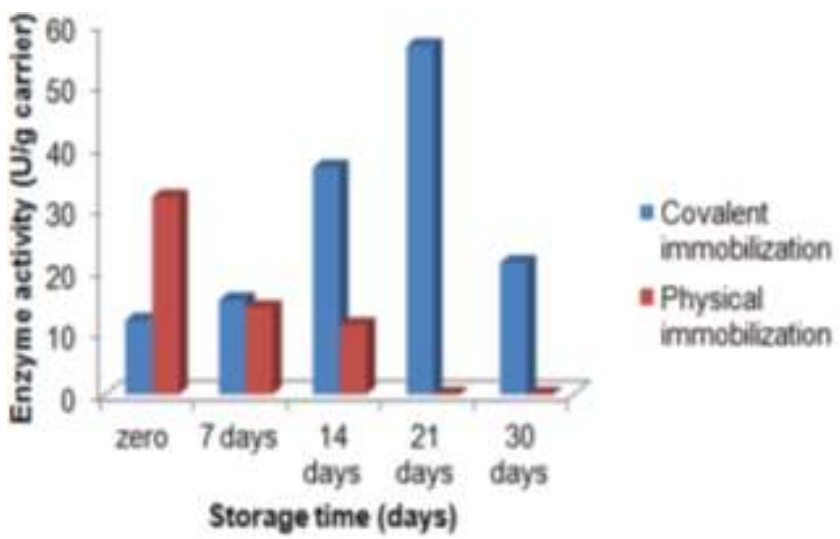

Fig. 2: Effect of storage time on immobilized L-ASNase activity onto fMWCNTs using covalent or physical immobilization techniques.

Table 1. Shows particle size distribution analysis and zeta potential of immobilized L-ASNase formulations (1) covalent and (2) physical) using DLS technique. The zeta potential of the prepared particles was increased from - $12.2 \mathrm{mv}$ in case of free MWCNTs relative to 36.3 and $14.2 \mathrm{mv}$ in case of immobilized MWCNTs using covalent and physical binding techniques respectively. The negative groups on the surface of L-ASNase were counteracted with the positively charged groups of MWCNTs-ANTA-Co ${ }^{2+}$ complex during the immobilization process. Moreover, some negative charges of the complex could counteract with the positively charged groups on the surface of L-ASNase and compact the enzyme both inside and on the surface of the particle. However, having a sufficient zeta potential is extremely important for the role of nanoparticles as carriers for bioactive compounds; the nanoparticles must be capable of ionically holding active molecules.

Table 1: Particle size distribution analysis and zeta potential of immobilized L-ASNase formulations (1) covalent and (2) physical) using DLS technique.

\begin{tabular}{|c|l|l|l|}
\hline Sample Code & $\begin{array}{l}\text { Particle size } \\
(\mathrm{nm})\end{array}$ & $\begin{array}{l}\text { Variance } \\
(\mathrm{PDI})\end{array}$ & Zeta potential (mv) \\
\hline L-ASNase & $667 \pm 117$ & 0.460 & $-2.7 \pm 10.5$ \\
\hline$(1)$ & $51 \pm 5.3$ & 1.0 & $36.3 \pm 6.0$ \\
\hline$(2)$ & $79 \pm 8.2$ & 1.0 & $14.2 \pm 3.7$ \\
\hline
\end{tabular}

Fig 3. Shows TEM morphologies and particle size analysis of the L-ASNase loaded fMWCNTs using (1) covalent and (2) physical techniques in comparison with the free MWCNTs. It was noticed that the dark core particles is due to the fact that the staining enzyme has penetrated through the tubes with average size $51 \pm 5.3$ and $79 \pm 8.2 \mathrm{~nm}$, PDI $\sim 1.0$, in case of samples (1) and (2), respectively. 


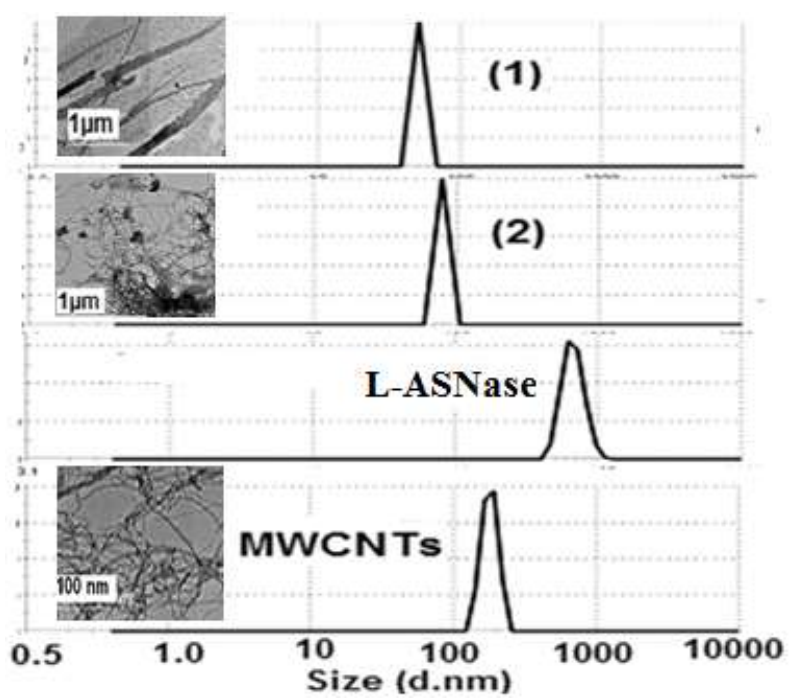

Fig. 3: Particle size distribution analysis of the immobilized L-ASNase formulations (1) covalent and (2) physical using DLS technique with different morphologies TEM images.

\section{Conclusion}

The results indicated that L-ASNase could be successful immobilized onto functionalized MWCNTs via covalent binding or physical adsorption techniques to enhance the storage ability at different temperatures and to minimize the lost in the enzyme activity. In other words, L-ASNase was produced from Aspergillus versicolor with max activity about $(45.53 \mathrm{U} / \mathrm{ml})$. The immobilized enzyme activity was gradually increased after storing for 3 weeks with particle size about $51 \mathrm{~nm}$, in case of the covalent technique relative to the physical one. Further study including in vivo biological examination using liver and kidney dysfunction biomarkers will be also investigated.

\section{Acknowledgements}

Authors acknowledge National research centre, Egypt, for financial support of this work under project No. 11090308.

\section{References}

[1] J. T. Cang-Rong, G. Pastorin, "The influence of carbon nanotubes on enzyme activity and structure: investigation of different immobilization procedures through enzyme kinetics and circular dichroism studies," Nanotechnol., vol. 20, pp. 255102, 2009.

[2] A. A. Haroun, F. A. Eman, M. A. Esawy, "Immobilization and characterization of levansucrase enzyme onto functionalized multi-walled carbon nanotubes," Egypt. J. Chem., vol. 61, pp. 667 - 678, 2018.

[3] A. A. Haroun, H. A Amin., S. H. Abd El-Alim, "Immobilization and in vitro evaluation of soyasapogenol B onto functionalized multi-walled carbon nanotubes," IRBM, vol. 39, pp. 35-42, 2018.

[4] W. Feng, P. Ji, "Enzymes immobilized on carbon nanotubes," Biotechnol Adv., vol. 29, pp. 889, 2011.

[5] L. Wang, R. Jiang, "Reversible his-tagged enzyme immobilization on functionalized carbon nanotubes as nanoscale biocatalyst," Methods Mol Biol., vol. 743, pp. 95, 2011.

[6] MW Marshall, S. Popa-Nita, JG. "Shapter Measurement of functionalized carbon nanotubes carboxylic acid groups using a simple chemical process," Carbon, vol. 44, pp. 1137, 2006.

[7] A. A. Haroun, H. A. Amin, S. H. Abd El-Alim, "Preparation, characterization and in vitro biological activity of soyasapogenol B loaded onto functionalized multi-walled carbon nanotubes," Current Bioactive Compounds, vol. 14, pp. 364-372, 2018.

[8] A. A. Haroun, A. H. Mossa, S. M. Mohafras, "Preparation and biochemical evaluation of functionalized multi-walled carbon nanotubes with P. granatum extract," Current Bioactive Compounds, vol. 15, pp. 138-144, 2019.

[9] R. K. Saxena, U. Sinha, "L-asparaginase and glutaminase activities in the culture filtrates of Aspergillus nidulans," Current Sci., vol. 50, pp. 218-219, 1981. 